\title{
Black-Scholes PDE: A Finance Application
}

\author{
Vuong Hoang Quan \\ Ecole de Commerce Solvay (Bruxelles)
}

International Conference on Differential Equations, Approximations and Applications, DEAA - 2001, pg. 53

\author{
December 10 - 15, 2001 \\ Vietnam National University \\ Hanoi, Vietnam
}

This is a collection of the abstracts of lectures given at the International Conference on Differential Equations, Approximations and Applications, which will be held at the old campus of the Vietnam National University at Hanoi December 10-15, 2001.

The Organizing Committee thanks the Vietnam National University at Hanoi, Hanoi University of Science, National Research Program for Basic Sciences, International Centre for Theoretical Physics, Mrs. G. Kumstadter, FPT Company for their generous financial supports. 


\section{INTERNATIONAL CONFERENCE on}

\section{DIFFERENTIAL EQUATIONS, APPROXIMATIONS and APPLICATIONS \\ DEAA - 2001}

December 10 - 15, 2001

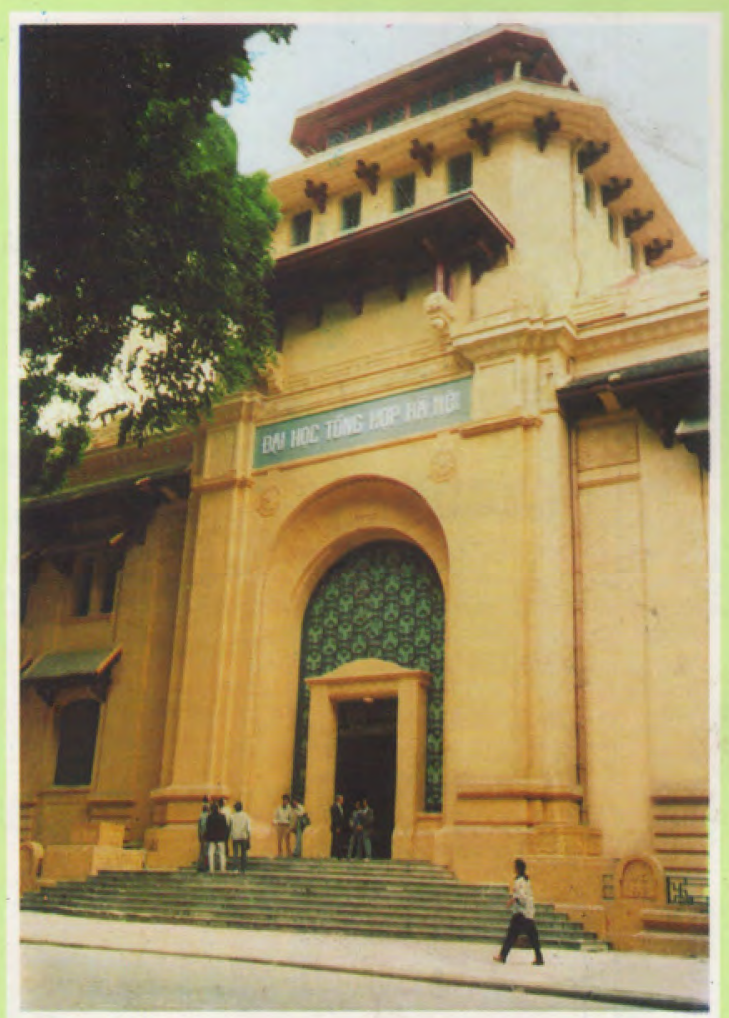

Vietnam National University

Hanoi, Vietnam 


\section{INTERNATIONAL CONFERENCE on}

\section{DIFFERENTIAL EQUATIONS, APPROXIMATIONS and APPLICATIONS \\ DEAA - 2001}

December 10 - 15, 2001

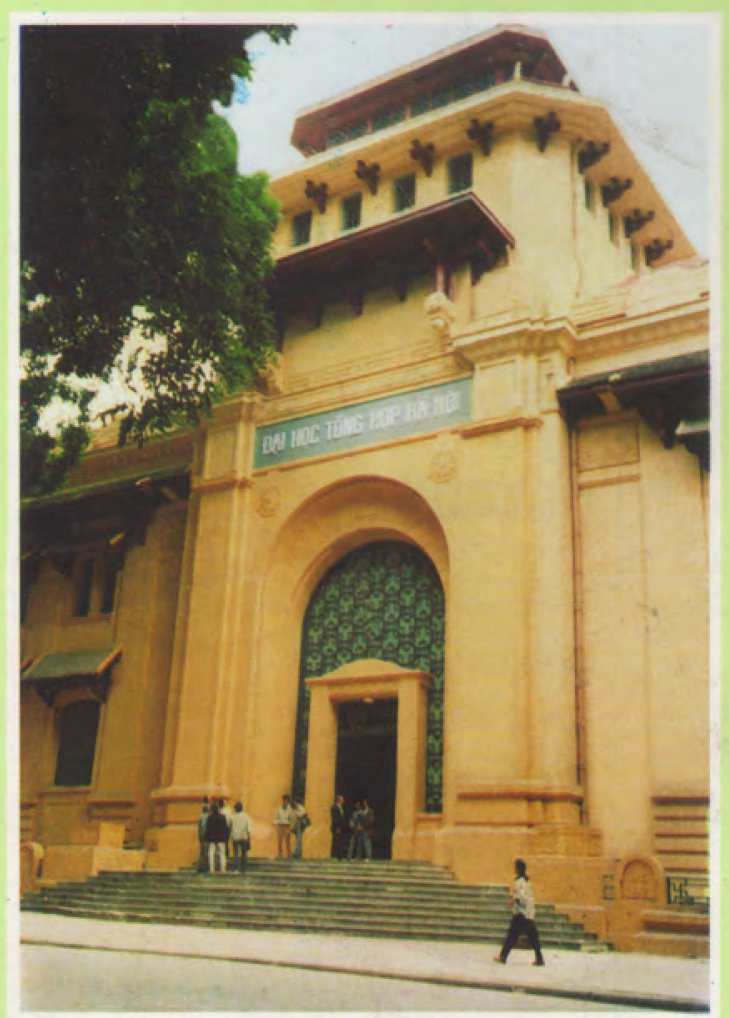

Vietnam National University

Hanoi, Vietnam 
This is a collection of the abstracts of lectures given at the International Conference on Differential Equations, Approximations and Applications, which will be held at the old campus of the Vietnam National University at Hanoi December 10-15, 2001.

The Organizing Committee thanks the Vietnam National University at Hanoi, Hanoi University of Science, $\mathrm{Na}$ tional Research Program for Basic Sciences, International Centre for Theoretical Physics, Mrs. G. Kumstadter, FPT Company for their generous financial supports. 


\section{Contents}

1 Plenary lectures

1.1. Stabilization of Dynamical Systems by bounded feedbacks ... 8

1.2. An Introduction to Validated Methods for Initial Value Problems for Ordinary Differential Equations . . . . . . . . . . . 9

1.3. Mean-square stability analysis of numerical schemes for stochastic differential equations . . . . . . . . . . . 10

1.4. Spectrum and Periodic Solutions of Functional Differential Equations

1.5. The operator pencil $(A-\lambda B)$ and asymptotic behavior of the degenerate equation $(B u)^{\prime}(t)=A u(t) \ldots \ldots \ldots \ldots \ldots \ldots \ldots \ldots \ldots$

1.6. New results on $q$-difference equalitions: Back to Birkhoff's program 13

1.7. Convergence rates of representation formulas for $M$-parameter semigroups . . . . . . . . . . . . . . . . . 14

1.8. Greedy Algorithms in Nonlinear Approximation ... . . . . 16

1.9. Optimal recovery and extremum theory . . . . . . . 17

1.10. Analytic continuation in some classes of separately analytic functions of real variables . . . . . . . . . . . . . 18

2 Section 1

2.1. On the asymptotics of solutions of the Cauchy problem in Banach spaces

2.2. Gevrey Formal Reduction of Vector Fields' Singularities .

2.3. Lyapunov spectrum of nonautonomous linear stochastic differential equations

2.4. Total Stability and the Existence of Almost Periodic Integrals for Almost Periodic General Processes ... . . . . . . . . . 24

2.5. Asymptotically Periodic Solutions of Volterra Difference Equations 26

2.6. Almost periodic solutions of higher order delay differential equations 27

2.7. Center Manifolds of Partial Functional Differential Equations Revisited . . . . . . . . . . . . . . . . . . 28

2.8. Asymtotic behavior of solutions of the system controlled by delayed feedback . . . . . . . . . . . . . . . . . . 29

2.9. Global Exponential Stability in Discrete-Time Analogues of Delayed Cellular Neutral Networks . . . . . . . . . . . . 30 


\subsection{Some Invariant Manifolds for Abstract Functional Differential}

Equations and Linearized Stabilities . . . . . . . . . . . . . 31

2.11. On the Complex WKB Analysis for a Second Order Linear O.D.E. of Schrödinger Type . . . . . . . . . . . . . . . . . . . . 32

2.12. Algebraic Solutions of Lotka-Volterra Equations . . . . . . . . . . . . 34

2.13. Symmetries, control and invariant tracking . . . . . . . . 35

2.14. Bounded Solutions and Periodic Solutions to Linear Differential Equations ......................... 36

2.15. About the applicability of the Ramis-Morales criteria for testing integrability of Hamiltonian systems . . . . . . . . . 37

3 Section 2

3.1. From differential-algebraic equations to implicit difference equations 40

3.2. Spectral conditions for bounded and almost periodic solutions of difference equations ................... . . . 41

3.3. Some classical inequalities for derivatives in Banach spaces generated by convex and concave functions . . . . . . . . . . . . . 42

3.4. Knowledge Discovery and Data Mining Approach to Computa-

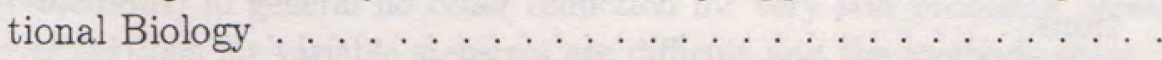

3.5. On the Stabilizability of Control Systems with Multiple Continuous Delays . . . . . . . . . . . . . . . . . . . . . . 4 4 . . . . . . 44

3.6. Generalized Collocation RKN Methods . . . . . . . . . . . 45

3.7. On the recursive sequence $x_{n+1}=\left(A x_{n}+B\right) /\left(x_{n}+a x_{n-1}+b\right) \ldots 46$

3.8. Regularization of a nonlinear integral equation of gravimetry . . . 47

3.9. Newmark's method and discrete energy applied to resistive MHDequation . . . . . . . . . . . . . . . . . . . . . . . 48

3.10. Surface temperature determination from Borehole measurement: Formulation as a moment problem ... . . . . . . . . . 49

3.11. The improved sweep method for solving nonself-adjoint boundary value problems ...................... 50

3.12. Some extensions and applications of Gronwall-Bellman's Lemma . 51

3.13. Krylov W-methods for large stiff ODE systems . . . . . . . . . . . 52

3.14. Black-Scholes PDE: A Finance Application . . . . . . . . . . . 53

3.15. Analysis of a Chemostat Model for Bacteria and Bacteriophage . 54

3.16. Fractals, Hausdorff dimension and Kakeya problem . . . . . . . 55

3.17. Permanence of host,parasitoid systems . . . . . . . . . . 56

3.18. Nonlinear Functional Integral Equation and Its Application . . . . 57 


\subsection{An Introduction to Validated Methods for Initial Value Problems for Ordinary Differential Equations}

\section{Ken Jackson}

\section{Computer Science Department University of Toronto Toronto, Canada, M5S 3G4 Email: krj@cs.toronto.edu}

Compared to standard numerical methods for initial value problems (IVPs) for ordinary differential equations (ODEs), validated methods have two important advantages: if they return a solution to a problem, then

1. the problem is guaranteed to have a unique solution, and

2. an enclosure of the true solution is produced.

We survey validated methods for the numerical solution of IVPs for ODEs, describe several methods in a common framework, and identify areas for future research.

This is joint work with Prof. Ned Nedialkov. 
CHAPTER 1

\section{PLENARY LECTURES}




\subsection{Stabilization of Dynamical Systems by bounded feedbacks}

R. Gabasov, F.M. Kirillova (Minsk, Belarus)

The stabilization is one of the actual problems of differential equations. It consists in constructing such the feedbacks that after closing properly unstable systems become asymptotically stable. A stabilization problem is essentially complicated if bounded feedbacks are used. Synthesis problems of stable dynamic systems invariant with respect to large exterior disturbances and robust with respect to large variations of systems parameters are of great importance. At solving the problems mentioned, methods of synthesis of optimal feedbacks turn out to be very effective. In the report a new approach to the solution of optimal synthesis problems is described. Linear, quasi-linear, piecewise-linear and nonlinear optimal control problems are under consideration. Results of computer experiments are given which demonstrate efficiency of algorithms suggested. Applications of methods of optimal synthesis for the solution of various stabilization problems are presented. Examples are given. 


\subsection{An Introduction to Validated Methods for Initial Value Problems for Ordinary Differential Equations}

\section{Ken Jackson}

\section{Computer Science Department \\ University of Toronto \\ Toronto, Canada, M5S 3G4 \\ Email: krj@cs.toronto.edu}

Compared to standard numerical methods for initial value problems (IVPs) for ordinary differential equations (ODEs), validated methods have two important advantages: if they return a solution to a problem, then

1. the problem is guaranteed to have a unique solution, and

2. an enclosure of the true solution is produced.

We survey validated methods for the numerical solution of IVPs for ODEs, describe several methods in a common framework, and identify areas for future research.

This is joint work with Prof. Ned Nedialkov. 


\title{
1.3. Mean-square stability analysis of numerical schemes for stochas- tic differential equations
}

\author{
Taketomo Mitsui \\ Graduate School of Human Informatics \\ Nagoya University \\ Nagoya 464-8601, Japan \\ tom.mitsui@cc.nagoya-u.ac.jp.
}

Stochastic differential equations (SDEs) represent physical phenomena dominated by stochastic processes. As for deterministic ordinary differential equations (ODEs), various numerical schemes have been proposed for SDEs under the principle of discrete variable methods. Numerical stability of the schemes turns out, then, to be more crucial for SDEs. We will describe the concept of the meansquare stability, and show its criteria for several schemes applied to scalar and vector SDEs.

This is a joint work with Y. Saito, Gifu Shotoku Gakuen University. 


\title{
1.4. Spectrum and Periodic Solutions of Functional Differential Equations
}

\author{
Toshiki Naito \\ The Univesity of Electo-Communications \\ Chofu, Tokyo 182-8585, Japan \\ naito@e-one.uec.ac.jp
}

In this talk, I will give an introduction to several results in the theory of functional differential equations. They are obtained by Naito and his colleagues; Nguyen Van Minh, Yoshiyuki Hino, Tetsuo Furumochi, Jong Son Shin, Satoru Murakami and others. We are interested in the study of the following equation:

$$
u^{\prime}(t)=A u(t)+F\left(t, u_{t}\right)+f(t)
$$

where $A$ is the infinitesimal generator of a $C_{0}$ semigroup on a Banach space $E$, $u_{t}$ is a function segment of $u$ at time $t$ defined as $u_{t}(\theta)=u(t+\theta),-\infty<\theta \leq 0$. The function $f(t)$ is continuous, peiriodic or almost periodic.

The function $F$ is assumed to be continuous in $\left(t, u_{t}\right)$, where $u_{t} \in \mathcal{B}$, a function space called the phase space. To deal with such delay equation systematically, Hale and Kato have defined an abstract phase space $\mathcal{B}$. It is a Banach space of some functions $\phi:(-\infty, 0] \rightarrow E$ satisfying natural axioms which are deduced from common properties of several concrete spaces appearing in the theory of delay equations. The important property is that $u_{t}$ moves continuously in the space $\mathcal{B}$ with respect to $t$ and $\left\|u_{t}\right\|_{B} \rightarrow 0$ as $t \rightarrow \infty$ provided $|u(t)|_{E} \rightarrow 0$ as $t \rightarrow \infty$ (the fading memory property). Assuming the fading memory property; we can deal with the equations with infinite delay as in the case of finite delay.

Among many results I will pick up followings ones: (i) the distribution of the spectrum of the generator of the solution semigroup on $\mathcal{B}$ of the linear autonomous equation and the stability of the solution:(ii) the existence of periodic solutions of linear periodic equation and the fixed point theorem by Chow and Hale;(iii) the method of commuting operators associated with the linear equation and the decomposition theorem;(iv) an introduction to the variation of constants formula and its application to the exisence of periodic and alomost periodic solutions; (v) the total stability of the equation and the existence of almost periodic solutions. 
1.5. The operator pencil $(A-\lambda B)$ and asymptotic behavior of the degenerate equation $(B u)^{\prime}(t)=A u(t)$

Vu Quoc Phong

Ohio University

We report on recent joint work with Sen-Yen Shaw. Of concern is the stability and almost periodicity of solutions of Eq. $(B u)^{\prime}(t)=A u(t)$, where $A$ and $B$ are closed linear operators from a Banach space $X$ to a Banach space $Y$. Our methods are based on the theory of linear operator pencil $(A-\lambda B)$ and on generalized Lyapunov-Sylvester equations $A X-B X D=C$. In particular, we obtain results on almost periodicity of solutions under the countability conditions of the imaginary spectrum of the pencil. 


\subsection{New results on $q$-difference equalitions: Back to Birkhoff's pro- gram}

\section{J-P. Ramis (France)}

$q$-difference equation is an old subject. They were studied by famous mathematicians: Euler, Gauss, Jacobi, Witten, Ramanujan, Birkhoff,...

As a differential equation is a relation between a function $f(x)$ and its derivatives: $f^{\prime}(x), f^{\prime \prime}(x), \ldots$ a $q$-difference equation (respectively, a difference equation) is a relation between $f$ and and its transformed by an automorphism on the variable: $f(q x), f\left(q^{2} x\right), \ldots$ (respectively, $f(x+1), f(x+2), \ldots$ ). Birkhoff began (in the thirties) to mimic for $q$-difference equations the work of Riemann and Hilbert for the differential equations, in particular to classify $q$-difference equations by "generic objects".

After a paper by Birkhoff and his student Guenter (1941) it seems that the subject was almost completely forgotten until quite recently. There is now a renewal (in relation in particular with $q$-combinatorics, quantum groups, theoretical physics,...).

I will explain how with my students J. Saulvy and C. Zhang, we completely archived Birkhoff's program on $q$-difference equations and deduce some fundamental results on $q$-difference Galois theory. I will explain in particular what is the " $q$-monodromy" and how one receives usual monodromy for linear O.D.E. when $q \rightarrow 1$. 


\title{
1.7. Convergence rates of representation formulas for $M$-parameter semigroups
}

\author{
Pei-Shan Huang and Sen-Yen Shaw \\ Department of Mathematics, National Central University \\ Chung-Li, Taiwan
}

Two kinds of $m$-dimensional versions of some well known approximation operators, such as those of Bernstein, Chung, Kantorovitch, Durrmeyer, and MeyerKönig-Zeller, are formulated. Then by applying a vector-valued version of a quantitative Korovkin approximation theorem of Shisha and Mond to them, we obtain estimates of convergence rates of some approximation formulas for a continuous vecter-valued function $f$ of $m$ variables, in terms of its modulus of continuity $\omega(f, \delta)$. This provides an approach to estimate convergence rates of some known or new representation formulas for a strongly continuous $m$-parameter semigroup $\left\{T(t)=T\left(t_{1}, t_{2}, \ldots, t_{m}\right) ; 0 \leq t_{i}<\infty, i=1,2, \ldots, m\right\}$ of operators on a Banach space $X$.

Denote $\bar{t}:=t_{1}+t_{2}+\cdots+t_{m}, \mathbb{T}:=\left\{t=\left(t_{1}, t_{2}, \ldots, t_{m}\right) ; 0 \leq t_{i} \leq 1, i=1, \ldots, m\right\}$, and $\tilde{\mathbb{T}}:=\left\{t=\left(t_{1}, t_{2}, \ldots, t_{m}\right) ; 0 \leq \bar{t} \leq 1\right\}$. Let $A_{i}$ be the infinitesimal generator of the $C_{0}$-semigroup $\left\{T_{i}\left(t_{i}\right):=T\left(0, \ldots, 0, t_{i}, 0, \ldots, 0\right) ; 0 \leq t_{i}<\infty\right\}, i=1,2, \ldots, m$. We have:

$$
\begin{aligned}
& \left\|\prod_{i=1}^{m}\left(I+t_{i}\left(T_{i}\left(\frac{1}{n}\right)-I\right)\right)^{n} x-T(t) x\right\| \leq 2 \omega(T(\cdot) x, \sqrt{\bar{t} / n}), x \in X, t \in \mathbb{T} ; \\
& \left\|\left(I+\sum_{i=1}^{m} t_{i}\left(T_{i}\left(\frac{1}{n}\right)-I\right)\right)^{n} x-T(t) x\right\| \leq 2 \omega(T(\cdot) x, \sqrt{\bar{t} / n}), x \in X, t \in \tilde{\mathbb{T}} ; \\
& \left\|\prod_{i=1}^{m}\left(I+t_{i}\left(\left(I-\frac{A_{i}}{n}\right)^{-1}-I\right)\right)^{n} x-T(t) x\right\| \leq 2 \omega(T(\cdot) x, \sqrt{2 \bar{t} / n}), x \in X, t \in \mathbb{T} \\
& \left\|\left(I+\sum_{i=1}^{m} t_{i}\left(\left(I-\frac{A_{i}}{n}\right)^{-1}-I\right)\right)^{n} x-T(t) x\right\| \leq 2 \omega(T(\cdot) x, \sqrt{2 \bar{t} / n}), x \in X, t \in \tilde{\mathbb{T}} \\
& \| \prod_{i=1}^{m}\left\{(n+1) A_{i}^{-1}\left(T_{i}\left(\frac{1}{n+1}\right)-I\right)\left(I+t_{i}\left(T_{i}\left(\frac{1}{n+1}-I\right)\right)^{n}\right\} x-T(t) x \|\right. \\
& \quad \leq 2 \omega\left(T(\cdot) x, \sqrt{\frac{(3 n-2) m}{3(n+1)^{2}}}\right), x \in X, t \in \mathbb{T} ;
\end{aligned}
$$




$$
\begin{aligned}
& \left\|\left(\prod_{i=1}^{m}(n+1) A_{i}^{-1}\left(T_{i}\left(\frac{1}{n+1}\right)-I\right)\right)\left(I+\sum_{i=1}^{m} t_{i}\left(T_{i}\left(\frac{1}{n+1}\right)-I\right)\right)^{n} x-T(t) x\right\| \\
& \leq 2 \omega\left(T(\cdot) x, \sqrt{\frac{(3 n-2) m}{3(n+1)^{2}}}\right), x \in X, t \in \tilde{\mathbb{T}}
\end{aligned}
$$




\title{
1.8. Greedy Algorithms in Nonlinear Approximation
}

\author{
V.N. Temlyakov
}

Our main interest in this talk is nonlinear approximation. The basic idea behind nonlinear approximation is that the elements used in the approximation do not come from a fixed linear space but are allowed to depend on the function being approximated. While the scope of the talk is mostly theoretical, we should note that this form of approximation appears in many numerical applications such as adaptive PDE solvers, compression of images and signals, statistical classification, and so on. The standard problem in this regard is the problem of $m$-term approximation where one fixes a basis and looks to approximate a target function by a linear combination of $m$ terms of the basis. When the basis is a wavelet basis or a basis of other waveforms, then this type of approximation is the starting point for compression algorithms. We will discuss the quantitative aspects of this type of approximation. We will also discuss stable algorithms for finding good or near best approximations using $m$ terms. These algorithms are representatives of a family of greedy algorithms. More recently, there has emerged another more complicated form of nonlinear approximation which we call highly nonlinear approximation. It takes many forms but has the basic ingredient that a basis is replaced by a larger system of functions that is usually redundant. Some types of approximation that fall into this general category are mathematical frames, adaptive pursuit (or greedy algorithms) and adaptive basis selection. Redundancy on the one hand offers much promise for greater efficiency in terms of approximation rate, but on the other hand gives rise to highly nontrivial theoretical and practical problems. We will discuss some of these theoretical problems in the talk. 


\subsection{Optimal recovery and extremum theory}

\section{Tikhomirov, Moscow}

The lecture is devoted to the problem of optimal recovery of linear functionals on classes of smooth, analytic functions and spaces of polynomials and harmonics. Recovery is one of the fundamental notions of the numerical analysis. It is possible to describe it as follows: to recover a mathematical object means to obtain a necessary data (maybe approximate), which is based on some information about the object (which as a rule is inexact).

Our considerations are based on some general principles of extremum. The main result of relationship of optimal recovery and Lagrange's multipliers of a certain convex extremal problem is applied to some concrete recovery problems. It leads to the generalization of many results in classical analysis, which date back to Tchebyshev, Zolotarev, A. and V. Markovs, Bernstein. Kolmogorov, Landau, Hardy-Littlwood-Polya, Favard and others.

In the lecture the following questions will be discussed:

1. Approximation of individual elements and problems of recovery.

2. Approximation by polynomials of individual functions and recovery based on values of certain moments.

3. Inequalities for derivatives of polynomials (algebraic, trigonometrical, spherical) and recovery of a value of a polynomial (or its derivative) in fixed points basing on inexact information.

4. Inequalities for derivatives of smooth functions and recovery of a value of a function (or its derivative) in a fixed point basing on inexact information.

5. Approximation and recovery of smooth and analitic functions from functional classes. 
1.10. Anabtic continuation in some classes of separately analytic functions of real variables

\section{Nguyen Thanh Van}

ULiversité de Paul Sabatier, Toulouse, France

For $j=: .2 \cdots m$, let $D_{j}$ be a domain in $\mathbb{R}^{N_{j}}, E_{j}=$ closure of a relatively conjaci oper ssbe: of $D_{j}$ with smooth boundary

$Q_{j}=$ a himoseneous polynomial of $N_{j}$ variables such that $Q_{j}(x)=0 \Rightarrow x=0$ E-1:

$$
\begin{aligned}
& \left.\because=\Xi^{\because}=\Xi_{2} \times \cdots \times E_{m}\right) \cup \cdots \cup\left(E_{1} \times \cdots \times E_{m-1} \times D_{m}\right) \\
& \because \because=\Xi \cdots \times E_{j-1} \times E_{j+1} \times \cdots E_{m}
\end{aligned}
$$

We :op: $: \because \because-C$ such that for every fixed $y \in Y_{j}$ the function $x \mapsto f(y, x)$ : I

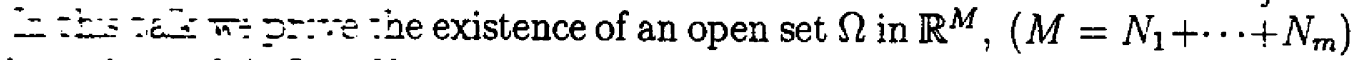
ind $0=:=z \therefore \Omega=\ldots$, such that $f$ has a real-analytic continuation on $\Omega$. 
CHAPTER 2

SECTION 1 


\title{
2.1. On the asymptotics of solutions of the Cauchy problem in Banach spaces
}

\author{
Pham Ngoc Boi \\ Department of Mathematics \\ University of Vinh
}

Let $\mathcal{B}$ be a Banach space, and let $A$ and $R(\varepsilon), \varepsilon \in\left[0, \varepsilon_{0}\right]$ be functions taking values in $[\mathcal{B}]$, the space of bounded linear operators acting on $\mathcal{B}$, integrable on $[0, T], T<+\infty$. In $\mathcal{B}$ we consider the following Cauchy problem

$$
\begin{aligned}
& \frac{d x(t)}{d t}=A(t) x(t), \quad x(0)=x_{0}, \\
& \frac{d x(t)}{d t}=[A(t)+R(t, \varepsilon)] x(t), \quad x(0)=x_{0} .
\end{aligned}
$$

The probiem of finding conditions for the convergence of of solutions of (2.1) to those of (2.2) in the case of Banach spaces has been investigated by many mathematicians, for instance. Kurzweil, Vorel, Antonsiewicz, Opial, Levin, Strauss, Yorke. Nguyen The Hoan. Zabreiko, Kostadinov, Nguyen Hong Thai,.... In this talk, we mill present several results in this direction. 


\subsection{Gevrey Formal Reduction of Vector Fields' Singularities}

Mireille Canalis-Durand

(joint work with Reinhard Schäfke)

We consider a Pfaffian form:

$$
\omega=f(x, y) d x-g(x, y) d y=0
$$

which satisfies $f(0,0)=g(0,0)=0$.

Here, we study nilpotent analytical form $\omega$ which are analytically equivalent to

$$
\omega_{1}=d\left(y^{2}-x^{3}\right)+\Delta(x, y)(2 x d y-3 y d x)
$$

where $\Delta(x, y)$ is a convergent series, $\Delta(0,0)=0$.

Such a differential form $\omega_{1}$ can be formally transformed into a final reduced form $([2])$ :

$$
\bar{\omega}=d\left(y^{2}-x^{3}\right)+\left(\Delta_{0}(h)+x \Delta_{1}(h)\right)(2 x d y-3 y d x)
$$

where $h=y^{2}-x^{3}, \Delta_{0}, \Delta_{1} \in \mathbb{C}[[h]]$. The form $\tilde{\omega}$ is unique if $\Delta_{1}(h)=1$, when $\Delta(x, y)=x+\ldots$.

We want to study the convergence/divergence of the series $\Delta_{0}$ and the nature of the change of variables $U(x, y)$ which transforms $\omega_{1}$ into $\tilde{\omega}$.

In ([1]), we conjectured that the series $\Delta_{0}(h)$ is divergent and has an optimal Gevrey order equal to 6 . We presented a "pattern recognition" algorithm for the growth rate of the coefficients of $\Delta_{0}$. Even the formal reduction of $d\left(y^{2}-x^{2}\right)+$ $\Delta(x, y)(2 x d y-2 y d x)$ was treated in order to show that Gevrey results agreed with the theory of J. Martinet and J.-P. Ramis ([3]).

In this talk, we show that $U$ and $\Delta_{0}$ are divergent in the generic case $(\Delta(x, y)=$ $x+\ldots)$. These series are Gevrey of order 1 in the homogeneous degree, i.e. if $U(x, y)=\sum b_{k l} x^{k} y^{l}$ and $\Delta_{0}=\sum B_{m} h^{m}$, then $\exists K, A>0 / \forall k, l, m$

$$
\begin{gathered}
\left|b_{k l}\right| \leq K A^{2 k+3 l}(2 k+3 l) ! \\
\left|B_{m}\right| \leq K A^{6 m}(6 m) !
\end{gathered}
$$

Moreover, these series are summable.

\section{REFERENCES}

[1] M. Canalis-Durand, F. Michel, M. Teisseyre, Algorithms for Formal Reduction of Vector Fields Singularities, Journal on Dynamical and Control Systems, vol. 7 , No. 1, (2001), 101-125. 
[2] F. Loray, Réduction formelle des singularitéscuspidales de champs de vecteurs analytiques, Differential Equations 158 1, (1999), 152-173.

[3] J. Martinet, J.-P. Ramis, Classification analytique des équations différentielles non linéaires résonnantes du premier ordre, Ann. Sci. Ec. Norm. Sup. 16 (1983), 571-621. 


\subsection{Lyapunov spectrum of nonautonomous linear stochastic differen- tial equations}

\section{Nguyen Dinh Cong}

Hanoi Institute of Mathematics

P. O. Box 631 Bo Ho, 10000 Hanoi, Vietnam.

Email address: ndcong@hanimath.ac.vn

We introduce a concept of Lyapunov exponents and Lyapunov spectram for nonautonomous linear stochastic differential equations. The Lyapunov exponents are defined samplewise via the two-parameter flow generated by the equation. We prove that Lyapunov exponents are finite and nonrandom. Lyapunov exponents are used for investigation of Lyapunov regularity and stability of nonautonomous stochastic differential equations. The results show that the concept of Lyapunov exponents is still very fruitful for stochastic objects and gives us a useful tool for investigating sample stability as well as qualitative behavior of nonautonomous linear and nonlinear stochastic differential equations.

Key words: Lyapunov exponents, Lyapunov spectrum, nonautonomous stochastic differential equation, two-parameter stochastic flow, Lyapunov regularity, stability.

MSC2000: Primary 60H10, 37H10, 34D08; secondary 60G17, 34F05, 93E15. 


\title{
2.4. Total Stability and the Existence of Almost Periodic Integrals for Almost Periodic General Processes
}

\author{
Y. Hino (Chiba, Japan)
}

The notion of processes introduced in $[2-3]$ and $[5-8]$ is a useful tool in the study of mathematical analysis for some phenomena whose dynamics is described by the equations which contains the derivative with respect time variable. Indeed, Dafermos [2], Hale [5] and the authors [6-7] derived some stability properties for processes and applied those to get stability results and the existence of almost periodic solutions for some kinds of equations, including functional differential equations, partial differential equations and evolut ion equations.

To ensure the existence of almost periodic solutions of almost periodic systems, the concept of total stability (for this definit ion, see [11]) of a bounded solution plays an important rule.

For general processes (equal to the concept of "general dynamical systems" in [1]), Bondi and Moauro [1] gave a definition of total stability of subset $M$ of a metric space $\mathcal{X}$ and proved uniform asymptotic stability of $M$ implies total stability of $M$. For example, this definition implies the usual definition of total stability of the constant solution for systems, when the unperturbed is generated by an ordinary differential equation in $R^{n}$ with the right hand side satisfies a Lipschitz condition. For details, s ee [1]. In this means, the concepts of the total stability of differential e quations and of general processes are slightly different.

In this talk, at first, we extend the concept of total stability of a subset $M$ of a metric space $\mathcal{X}$ given by Bondi and Moauro to the concept of the total stability of a bounded integral $\mu(t)$ of a general process $w(t, s, x)$ on a metric space $\mathcal{X}$ and show that the total stability of an integral $\mu(t)$ of an almost periodic general process $w(t, s, x)$ implies the existence of an almost periodic integral of the general process $w(t, s, x)$. Secondly, we show that uniform asymptotic stability of an integral $\mu(t)$ of a (not general) process $w(t, s, x)$ implies total stability of an integral $\mu(t)$. By Kato and Sibuya's example [9], it is known that the above result does not hold even for almost periodic general prosesses. Finally, for a linear process, we show that total stability of the equilibrium point zero implies uniform asymptotic stability of the equilibrium point zero, which corresponds to Massera's theorem [10] for ordinary differential equations.

\section{REFERENCES}

[1] P. Bondi and V. Moauro, Total stability for general dynamical systems, Ricerche di Matematica 25 (1976), 163-175.

[2] C. M. Dafermos, An invariance principle for compact processes,

J. Differential Equations 9 (1971), 239-252.

[3] C. M. Dafermos. Almost periodic processes and almost periodic solutions of crolution equations. Proceedings of a Liniversity of Florida Int ernational 
Symposium, Academic Press, New York, 1977, 43-57.

[4] J. K. Hale, Theory of Functional Differential Equations, Applied Math. Sciences 3, Springer-Verlag, New York, 1977.

[5] J. K. Hale, Asymptotic Behavior of Dissipative Systems, American Mathematical Society, Providence, Rhode Island, 1988.

[6] Y. Hino and S. Murakami, A genelarization of processes snd stabilities in abstract functional differential equations, Funkcial. Ekvac., 41 (1998), 235-255.

[7] Y. Hino and S. Murakami, Almost periodic processes and the existence of almost periodic solutions Electronic J. of QTDE, 3, (1998), 1-19.

[8] Y. Hino, T. Naito, N. Minh and J-S. Shin, Almost Periodic Solutions of Differential Equations in Banach space, Taylor and Francis, 2001.

[9] J. Kato and Y. Sibuya, Catastrophic deformation of a flow and non-existence of almost periodic solutions J. Fac. Sci. Univ. Tokyo Sect. IA Math. 24, (1977), 267-279.

[10] J. L. Massera, Contributions to stability theory, Ann. of Mat., 64 (1956), 182-206.

[11] T. Yoshizawa, Stability Theory and the Existence of Periodic Solutions and Almost Periodic Solutions, Springer-verlag, New York, Heidelberg, Berlin, 1975. 
2.5. Asymptotically Periodic Solutions of Volterra Difference Equations

\section{Tetsuo Furumochi}

Department of Mathematics, Shimane University, Matsue 690-8504, JAPAN

In this talk, we consider the Volterra difference equations

$$
x_{n+1}=a(n)-\sum_{k=0}^{n} D\left(n, k, x_{k}\right), \quad n \in Z^{+}
$$

and

$$
x_{n+1}=p(n)-\sum_{k=-\infty}^{n} P\left(n, k, x_{k}\right), \quad n \in Z,
$$

where $Z^{+}$and $Z$ denote the sets of nonnegative integers and integers respectively, $p$ is $N$-periodic, $P(n+N, k+N, x)=P(n, k, x), D(n, k, x)$ and $P(n, k, x)$ are at least continuous in $x$, and where $a(n)$ converges to $p(n)$ and $D(n, k, x)$ converges to $P(n, k, x)$ in some sense. We discuss periodicity and asymptotic periodicity by using Schauder's first theorem and a growth condition on $P$, and relations betwees solutions of the two equations. 


\title{
2.6. Almost periodic solutions of higher order delay differential equa-
} tions

\author{
Ha Binh Minh \\ Department of Applied Mathematics \\ Hanoi University of Technology \\ Dai Co Viet Street, Hanoi, Vietnam
}

We consider the following equation:

$$
A_{0} u(t)+\sum_{i=1}^{n} A_{i} u^{(i)}(t)+\sum_{j=0}^{m} \int_{a}^{b} d B_{j}(\eta) u^{(j)}(t+\eta)=f(t) .
$$

Here $A_{0}$ is a linear closed operator on the complex space $\mathbb{X} ; A_{i} \in L(\mathbb{X}), \forall i=$ $1 \ldots n ; B_{j} \in B V([a, b], L(\mathbb{X})), \forall j=0 \ldots m$. After introducing the definition of spectrum of Eq. (*) we investigate the almost periodicity of mild solutions of $(*)$ based on the countability of the imaginary spectrum of Eq. $(*)$ with $f$ almost periodic. The obtained results extend some previous ones to the above mentioned class of equations and can be further extended to various spaces of functions, which are called $\Lambda$-classes.

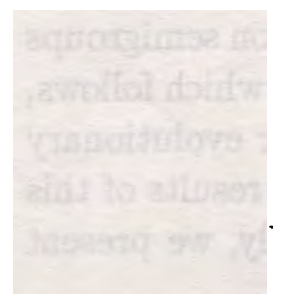




\title{
2.7. Center Manifolds of Partial Functional Differential Equations Re- visited
}

\author{
Nguyen Van Minh \\ Department of Mathematics, \\ Hanoi Unversity of Science, \\ Khoa Toan, DH Khoa Hoc Tu Nhien, \\ 334 Nguyen Trai, Hanoi, Vietnam.
}

We consider partial functional differential equations in the abstract form

$$
\dot{x}(t)=A x+F x_{t}+g\left(x_{t}\right)
$$

where $A$ is the generator of a $C_{0}$-semigroup of linear operators on a Banach space $\mathbb{X}, F \in L(\mathcal{C} ; \mathbb{X})$ and $g \in C^{1}(\mathcal{C}, \mathbb{X})$ satisfies $g(0)=0, D g(0)=0,\|g(\varphi)-g(\psi)\| \leq$ $L\|\varphi-\psi\|, \forall \varphi, \psi \in \mathcal{C}:=C([-r, 0], \mathbb{X})$. As is well known, if $A$ generates a compact semigroup, then the linear equation

$$
\dot{x}(t)=A x(t)+F x_{t}
$$

generates an eventually compact semigroup, so this semigroup has an exponential trichotomy (see the definition in Section 2 below). That for "sufficiently small" $g$ Eq. (2.3) has invariant manifolds has been considered in various papers. The existence of center manifolds for Eq. (2.3) plays an important role in the theory of bifurcations, however, the proof of the existence of center manifolds for Eq. (2.3) has been based on the so-called "variation-of-constants formula" in the phase space $\mathcal{C}$ of Memory. As is noted in our previous papers, the validity of this formula in general is stil open. The reason for this is due to the lack of Riesz representation of a continuous functional in infinite dimensional Banach spaces.

In the present paper we will make an attempt to fill this gap by giving a proof of the existence of center manifolds for Eq. (2.3) without using the variation-ofconstants formula in the phase space $\mathcal{C}$. Instead of using the variation-of-constants formula in the phase space, which is known as the Lyapunov-Perron method, we will employ the method of graph transforms. By this method we can include into our consideration a large class of equations which may generate non strongly continuous evolutionary processes. We now outline the contents of this paper. In the next section we will collect some well known results on the solution semigroups associated with Eq. (2.3) for the reader's convenience. In the section which follows, we present a method to prove the existence of center manifolds for evolutionary processes which are not necessarily strongly continuous. The main results of this paper will be based on the ones for evolutionary processes. Finally, we present several examples to illustrate the obtained results.

This is a join work with J. Wu (York University, Canada). 
sq.red

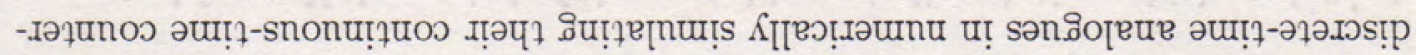

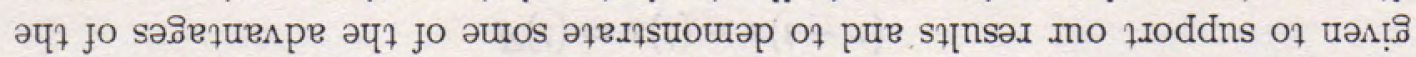

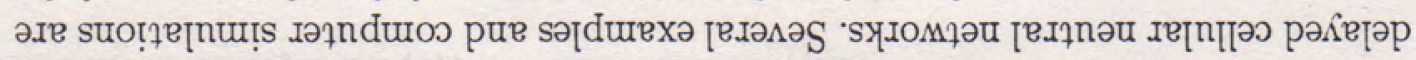

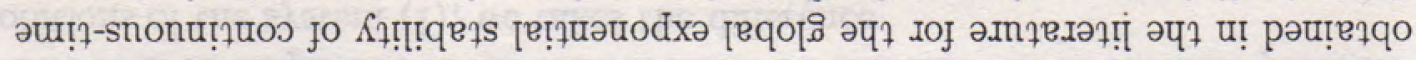

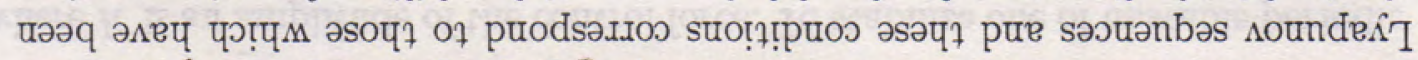

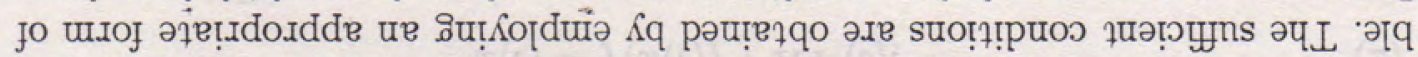

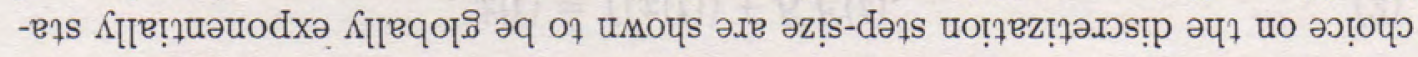

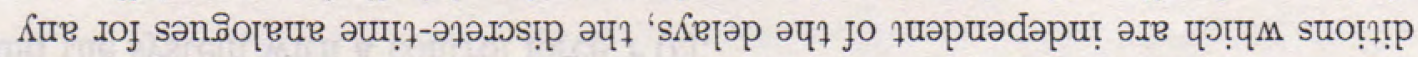

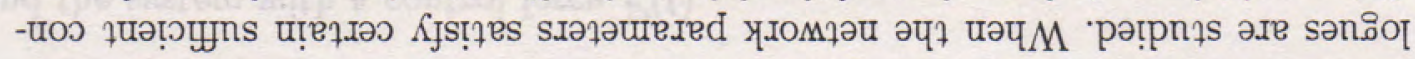

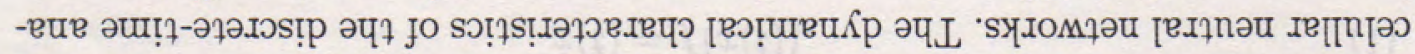

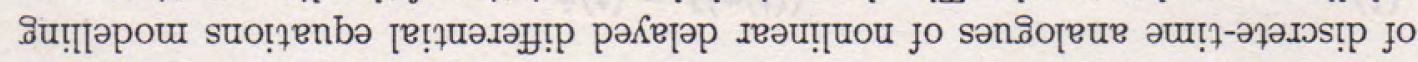

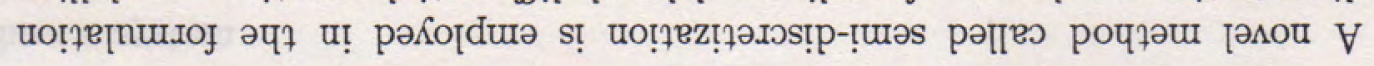

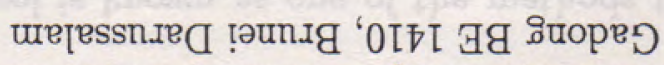

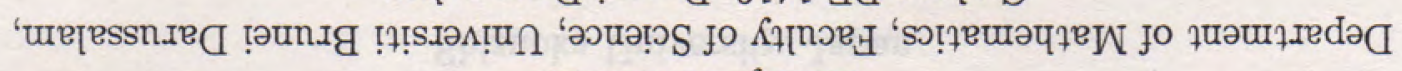

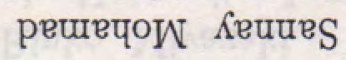




\title{
2.9. Global Exponential Stability in Discrete-Time Analogues of De- layed Cellular Neutral Networks
}

\author{
Sannay Mohamad \\ Department of Mathematics, Faculty of Science, Universiti Brunei Darussalam, \\ Gadong BE 1410, Brunei Darussalam
}

A novel method called semi-discretization is employed in the formulation of discrete-time analogues of nonlinear delayed differential equations modelling celullar neutral networks. The dynamical characteristics of the discrete-time analogues are studied. When the network parameters satisfy certain sufficient conditions which are independent of the delays, the discrete-time analogues for any choice on the discretization step-size are shown to be globally exponentially stable. The sufficient conditions are obtained by employing an appropriate form of Lyapunov sequences and these conditions correspond to those which have been obtained in the literature for the global exponential stability of continuous-time delayed cellular neutral networks. Several examples and computer simulations are given to support our results and to demonstrate some of the advantages of the discrete-time analogues in numerically simulating their continuous-time counterparts. 


\title{
2.10. Some Invariant Manifolds for Abstract Functional Differential Equations and Linearized Stabilities
}

\author{
Satoru Murakami \\ Department of Applied Mathematics, \\ Okayama University of Science, \\ 1-1 Ridaicho, Okayama 700-0005, Japan \\ Email: murakami@youhei.xmath.ous.ac.jp
}

In this talk, we will be concerned with the abstract functional differential equation

$$
\dot{u}(t)=A u(t)+L\left(u_{t}\right)+f\left(u_{t}\right)
$$

where $A$ is the infinitesimal generator of a strongly continuous compact semigroup on a Banach space $X, u_{t}$ is an element of $\mathcal{B}$ defined by $u_{t}(\theta)=u(t+\theta)$ for $\theta \in(-\infty, 0], L: \mathcal{B} \mapsto X$ is a bounded linear operator and $f \in C^{1}(\mathcal{B} ; X)$ with $f(0)=f^{\prime}(0)=0$; here $\mathcal{B}=\mathcal{B}((-\infty, 0] ; X)$ is the phase space for Eq. (1) which satisfies some fundamental axioms.

The main purpose of this talk is to prove the existence of some invariant manifolds for Eq. (1) such as stable manifold and unstable manifold, by using the variation-of-constants formula in the phase space for Eq. (1) which has recently been established by Hino, Murakami, Naito and Nguyen V. Minh in the paper [A variation-of-constants formula for abstract functional differential equations in the phase space, J. Differential Equations (in press)].

As a corollary of our main result, one can get a stability result on the zero solution of Eq. (1) which is often called as the principle of linearized stability in the theory of ODEs; indeed, the zero solution of Eq. (1) is uniformly asymptotically stable if the zero solution of the linearized equation

$$
\dot{u}(t)=A u(t)+L\left(u_{t}\right)
$$

is uniformly asymptotically stable.

Furthermore, establishing the existence of the center-unstable manifold for Eq. (1), an instability result on the zero solution of Eq. (1) is derived under the situation that the characterisitic operator for Eq. (2) possesses a characteristic root with positive real part. 
2.11. On the Complex WKB Analysis for a Second Order Linear O.D.E. of Schrödinger Type

\section{Minoru Nakano}

Department of Mathematics, KEIO University, 3-14-1 Hiyoshi, Kohoku, Yokohama, Japan, 223-8522.

Tel: +81 45566 1666. Fax: +81 45566 1642. E-mail: nakano@math.keio.ac.jp

We study the following second order linear ordinary differential equation of : Schrödinger type containing a small parameter $\varepsilon$, which sometimes represents the Planck constant:

$$
\begin{gathered}
\varepsilon^{2 h} \frac{d^{2} y}{d x^{2}}-a(x, \varepsilon) y=0 \quad\left(h \in \mathbb{N} ; x, y \in \mathbb{C} ; 0 \leq|x| \leq x_{0} ; 0<\varepsilon \leq \varepsilon_{0}\right), \\
a(x, \varepsilon):=\sum_{j=0}^{h^{-}} a_{j} x^{m_{j}} \varepsilon^{j} \quad\left(a_{0} \neq 0 ; a_{j} \in \mathbb{C} ; m_{0}>0\right),
\end{gathered}
$$

where $x_{0}, \varepsilon_{0}$ are small constants and $m_{j}$ 's are integers. A zero $x=0$ of $a(x, 0)(=$ $\left.a_{0} x^{m_{0}}\right)$ is called a turning point of (1).

Our aim is to get asymptotic property of solutions of (1) near $x=0$ when $\varepsilon \rightarrow 0$ by using the concept of the characteristic polygon for (1) introduced in Iwano-Sibuya [3] and by applying the so-called stretching-matching method and the complex WKB method (Nakano [4], [5], Nishimoto [6], Wasow [10]).

We plot the following points on the $(X, Y)$-plane according to the indexes of $\varepsilon$ and $x$ of $a(x, \varepsilon)$ :

$$
P_{j}:=\left(\frac{j}{2}, \frac{m_{j}}{2}\right) \quad(j=0,1,2, \cdots, h), \quad R:=(h,-1) .
$$

The characteristic polygon for (1) is defined to be a polygon convex downward connecting the points $P_{j}$ 's and $R$, then it consists of several segments. The more segments, the more complicated to analyze (1).

The simplest is the Airy equation

$$
\varepsilon^{2} y^{\prime \prime}-x y=0,
$$

whose characteristic polygon consists of one segment. The second simplest is

$$
\varepsilon^{2} y^{\prime \prime}-\left(x^{m}+\varepsilon x^{n}\right) y=0
$$

with a two-segment characteristic polygon (Nakano [4], Roos [8]). Roos also analyzes the equation with a three-segment characteristic polygon (Roos [9]), which is

$$
\varepsilon^{4} \frac{d^{2} y}{d x^{2}}-\left(x^{5}+\varepsilon x^{2}+\varepsilon^{2}\right) y=0
$$

In this talk. we consider (1) with an arbitrarily many-segment characteristic polygon. 


\section{References}

[1] Evgrafov, M. A. and M. V. Fedoryuk, Asymptotic behavior as $\lambda \rightarrow \infty$ of solutions of the equation $w^{\prime \prime}(z)-p(z, \lambda) w(z)=0$ in the complex $z$-plane. Uspehi Mat. Nauk 21, or Russian Math. Surveys 21 (1966), 1-48.

[2] Fedoryuk, M. V., Asymptotic Analysis. Springer Verlag (1993).

(English translation of "Федорюк, М.В., Асимптотические методы для линеңных обыкновенных дифференциалных уравнение. Наука (1983).”)

[3] Iwano, M. and Y. Sibuya, Reduction of the order of a linear ordinary differential equation containing a small parameter. Kodai Math. Sem. Rep. 15 (1963), 1-28.

[4] Nakano, M., On asymptotic solutions of a second order linear ordinary differential equation with a turning point I, II. Bull. of Hiyoshi. 14 (1972), 70-75. 15 (1973), 64-70.

[5] - On a $n$-th order linear ordinary differential equation with a turningsingular point. Tokyo J. Math. 21 (1998), 201-215.

[6] Nishimoto, T., On matching method for a linear ordinary differential equation containing a parameter I, II, III. Kodai Math. Sem. Rep. 17 (1965), 307-328; 18 (1966), $61-86 ; 19$ (1967), 80-94.

[7] Olver, F. W. J., Asymptotics and special functions. Academic Press (1974).

[8] Roos, H. G., Die asymptotische Lösung einer linearen Differentialgleichung zweiter Ordnung mit zweisegmentigem charakteristischem Polygon. Beitr. Anal. 7 (1975), 55-63.

[9] —_- Die asymptotische Lösung einer linearen Differentialgleichung mit dreisegmentigem charakteristischem Polygon. Math. Nachr. 88 (1979), 93-103.

[10] Wasow, W., Turning point problems for systems of linear differential equations, I. The formal theory, II. The analytic theory. Comm. Pure Appl. Math. 14 (1961), 657-673. 15 (1962), 173-187.

[11] — Linear turning point theory. Springer-Verlag (1985). 


\title{
2.12. Algebraic Solutions of Lotka-Volterra Equations
}

\author{
Kenzi Odani \\ Department of Mathematics, Aichi University of Education \\ Igaya-cho, Kariya-shi 448-8542, Japan
}

In the paper, we consider the condition of the Lotka-Volterra equation

$$
\dot{x}=x\left(b_{1} x-\left(a_{2}+b_{2}\right) y+a_{3}\right), \quad \dot{y}=y\left(\left(a_{1}+b_{1}\right) x-a_{2} y-b_{3}\right) .
$$

to have an invariant algebraic curve. We say that an algebaic curve is invariant under (LV) if it consists of some solutions of (LV). Both the lines $x=0$ and $y=0$ are anytime invariant under (LV). So we call them the trivial invariant lines of (LV).

If $a_{1} a_{2} a_{3}=b_{1} b_{2} b_{3}=0$, then we can integrate the equation (LV) by the separation of variables. If $a_{1} a_{2} a_{3} \neq 0$ and $b_{1} b_{2} b_{3}=0$, then we exchange $\left\{a_{i}\right\}$ and $\left\{b_{i}\right\}$ by changing the variables $(x, y, t) \rightarrow(y, x,-t)$. So the assumption $b_{1} b_{2} b_{3} \neq 0$ does not spoil the generality of the coefficients. From now on, we use such the notations as $k:=a_{1} / b_{1}$, $\ell:=a_{2} / b_{2}, m:=a_{3} / b_{3}$. The author obtains the following results:

Theorem 1. When $b_{1} b_{2} b_{3} \neq 0$, the equation (LV) has an invariant algebraic curves of degree $\leqq 2$ other than trivial ones if and only if it satisfies one of the following:

(1) $k \ell m=1$. (2.1) $k=-1$. (2.2) $\ell=-1$. (2.3) $m=-1$.

(3) $\quad k \ell m=-1, k+1 / m=1$. (4.1) $k=-2, m+1 / \ell=1$.

(4.2) $\quad \ell=-2, \quad k+1 / m=1 . \quad$ (4.3) $\quad m=-2, \quad \ell+1 / k=1$.

(5.1) $k=-1 / 2, \quad m+1 / \ell=1 . \quad(5.2) \quad \ell=-1 / 2, \quad k+1 / m=1$.

(5.3) $m=-1 / 2, \quad \ell+1 / k=1$.

If the equation (LV) satisfies one of (1), (2.1), (2.2), (2.3), then it has an invariant line other than trivial ones.

Theorem 2. When $b_{1} b_{2} b_{3} \neq 0$ and when all $k, \ell, m$ are not negative rationals, if the equation (LV) has an invariant algebraic curves other than trivial ones, then it satisfies the condition (1) or (3) of Theorem 1.

Actually, Theorem 1 is not new. In fact, Cairó, Feix and Llibre [1] already gave an essentially same result, however, not sophisticated. Our notation makes their result more clear. From Theorem 2, the author predicts that there are only a few number of irreducible invariant algebraic curves of degree $\geqq 3$.

\section{References}

1. L. Cairó, M. R. Feix, J. Llibre, Darboux method and search of invariants for the Lotka-Volterra and Complex quadratic systems, J. Math. Phys. 40 (1999), 2074-2091. 


\subsection{Symmetries, control and invariant tracking}

Pierre Rouchon

Ecole des Mines de Paris

E-mail: rouchon@cas.ensmp.fr

The talk is relative to output tracking $y=h(x)$ of a control system $\dot{x}=f(x, u)$. Tracking a reference trajectory $t \mapsto y_{r}(t)$ via classical decoupling and input/ouput linearization techniques provides a linear and stable closed-loop dynamics for the tracking error $e=y-y_{r}$. For many physical systems, the intrinsic character of $y-y_{r}$ is not guaranty. It is well known that when $y \in S O(3)$ is the orientation of a satellite, the "good" tracking error is $y\left(y_{r}\right)^{-1}$. When $y$ is the outlet concentration of a chemical reactor, the case is less classical. $y$ belongs to the simplex of molar fractions (each component lies in $[0,1]$ and the sum is 1 ). The difference $y-y_{r}$ has no physical meaning. One has to measure the tracking error in a different way in order to be invariant with respect to units changes: the tracking controller must remain unchanged if instead of using mole fractions we use mass fractions. Such natural invariance properties can be ensured if we can have an invariant way to measure the tracking error. For a general system admitting a symmetry group (up to static feedback), it is possible to derive (under some regularity assumptions relative to the group action) a collection of independent invariants, functions of $y$ and $\left.y_{r}, \ldots y_{r}^{(} \nu\right)$ for some derivation order $\nu$ measuring the tracking error. The construction of such invariants is based on the Darboux-Cartan moving frame method. Several examples of invariant errors will be given (chemical reactor and units changes, non holonomic systems with $S E(2)$ invariance, mechanical system with Galilean invariance, ... ). Application of input/ouput linearization techniques with these invariant errors as outputs automatically yields to invariant tracking controllers. 


\title{
2.14. Bounded Solutions and Periodic Solutions to Linear Differential Equations
}

\author{
Toshiki Naito \\ The Univesity of Electo-Communications \\ Chofu, Tokyo 182-8585, Japan \\ naito@e-one.uec.ac.jp \\ Jong Son Shin \\ Korea University \\ Kodaira, Tokyo 187-8560, Japan \\ shinjs@tech.korea-u.ac.jp
}

In this talk, we will give criteria on the existence of bounded solutions and periodic solutions to the equation of the form

$$
\frac{d}{d t} u(t)=A u(t)+f(t)
$$

where $A$ is the infinitesimal generator of a $C_{0}$-semigroup $U(t)$ on a Banach space $\mathrm{X}$, and $f(t)$ is a $\tau$-periodic continuous function.

Roughly speaking, the existence of periodic solutions to Equation (2.7) is derived from the existence of bounded solutions on $[0, \infty)$. It is called the Massera type theorem.

The existence of bounded solutions is, in the abstract form, represented as follows : there is an $x_{0}$ such that $\left\{V^{n} x_{0}\right\}_{n=1}^{\infty}$ is bounded for an affine linear map $V x=T x+b$, where $T$ is a bounded linear operator on $\mathrm{X}$ and $b(\neq 0) \in \mathrm{X}$ is fixed.

Our manner is based on the following fact : if

$$
\limsup _{n \rightarrow \infty}\left\|\sum_{k=0}^{n-1} T^{k} b\right\|<\infty
$$

then $\left\{V^{n} b\right\}_{n=0}^{\infty}$ is bounded, because $V^{n} b=\sum_{k=0}^{n} T^{k} b, n \in \mathbb{N}$. The relation (2.8) in Equation (2.7) becomes the following one :

$$
\limsup _{n \rightarrow \infty}\left\|\sum_{k=0}^{n-1} U(k \tau) \int_{0}^{\tau} u(\tau-s) f(s) d s\right\|<\infty .
$$

Hence, to obtain criteria on the existence of bounded solutions to Equation (2.7), we will employ the above relation (2.9).

This manner can be applied to evolutionary systems and functional differential equations. 
2.15. About the applicability of the Ramis-Morales criteria for testing integrability of Hamiltonian systems

\section{Jacques-Arthur Weil}

This is joint work with Delphine BOUCHER (Univ. Rennes 1, France)

A Hamiltonian system with $n$ degrees of freedom is said to be completely integrable if it admits $n$ first integrals in involution. Building on beautiful work of Ziglin, Ramis and Morales gave a powerful criterion for testing the (non)-complete integrability of a Hamiltonian system : given some solution curve $\Gamma$ (with mild conditions), the Hamiltonian system admits $n$ first integrals which are meromorphic in a neighborhood of $\Gamma$ and in involution only if the differential Galois Group of the variational equation(s) along $\Gamma$ has an abelian Lie algebra.

However, this property is still abstract and requires some skill (and or heavy computations) to be detected. In this work, we present several practical caracterizations for this property, based on the local study of linear differential equations, their factorization, and the smart use of a computer. Our method are specifically adapted to the case of families of equations depending on parameters. 
38

CHAPTER 2. SECTION 1

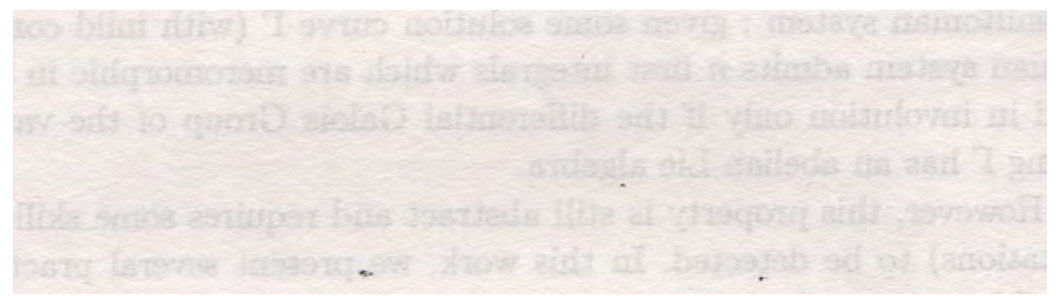

Digitized by Google 
CHAPTER 3

SECTION 2 


\subsection{From differential-algebraic equations to implicit difference equations}

\section{By Pham Ky Anh, Nguyen Huu Du and Le Cong Loi \\ Vietnam National University, Hanoi}

This report slimmarizes our recent results on implicit difference equations (IDEs):

$$
A_{n} x_{n+1}+B_{n} x_{n}=q_{n}
$$

where $A_{n}, B_{n} \equiv \mathbb{R}^{m \times n}, q_{n} \in \mathbb{R}^{m}$ and the matrices $A_{n}$ are all singular.

1. A notior of index-1 linear IDEs based on singular value decompositions (SVDs) of $A_{n}$ has bee- introduced. It is proved that the index of linear IDEs does not depend on the chosen SVDs of $A_{n}$. The unique solvability of some initial value problems (IVPs) for incex-1 linear has been established,

2. Tnder certain additional conditions, the solvability of the mentioned above IVPs fo: linear IDE $\leqslant$. winere $\operatorname{ker} A_{n+1} \subseteq \operatorname{ker} A_{n}$ or $\operatorname{rank} A_{n+1} \geq \operatorname{rank} A_{n}$ for all $n$, has been investigated.

3. The mu:-ipoint boundary-value problems (MPBVPs)

$$
\begin{aligned}
& A_{n} x_{n+1}+B_{n} x_{n}=q_{n} \quad(n=\overline{0, N-1}) \\
& \sum_{n=0}^{N} C_{n} x_{n}=\gamma
\end{aligned}
$$

where $\mathrm{N}$ becomes iarge, represents a large-scale system of linear equations. If the linear IDE (3.2) of irdex-1, a regular condition for MPBVPs (3.2), (3.3) has been introduced. It has been shown that the regularity of an MPBVP is a necessary and sufficient condition fo: a uniçue solvability of (3.2), (3.3). Another necessary and sufficient condition fo: the solvabiity of (3.2), (3.3) when the regularity condition does not hold has been establishec. Tzus combining the results in both regular and irregular cases, we arrive at a Fredhoim alternative for a special large scale system of linear equations (3.2), (3.3)

4. A connection between linear transferable DAEs and linear index-1 IDEs has been revealed. It is proved that the explicit Euler method applied to linear transferable DAEs leads to linear index-1 IDEs. Moreover, the solution of an IVP for linear index-1 IDEs, obtained by the explicit Euler method converges to the solution of the corresponding IVP for linear :ransferable DAEs 
3.2. Spectral conditions for bounded and almost periodic solutions of difference equations

\author{
Phi Thi Van Anh \\ Hanoi University of Science
}

We investigate spectral conditions for the following equation

$$
A x_{n+1}=B x_{n}+f_{n}, \quad n \in \mathbb{Z},
$$

to have bounded, almost periodic solutions. Here $\left\{x_{n}\right\},\left\{f_{n}\right\}$ are sequences in a Banach space $\mathbb{X}, A$ is a bounded linear operator acting on it. Using the notion of spectra of bounded sequences the following results are obtained.

Theorem 3.1. Let $\Lambda$ be a closed subset of the unit circle and let $\Lambda(\mathbb{X})$ be the space of all sequences with spectrra contained in $\Lambda$. Furthermore, assume that $A, B$ are commutative operators such that

$$
\sigma(A) . \Lambda \cap \sigma(B)=\emptyset
$$

Then for every $f \in \Lambda(\mathbb{X})$ there exists a unique $x_{f} \in \Lambda(\mathbb{X})$ as a solution (3.4).

Corollary 3.1 In Eq. (3.4) if

$$
\sigma(A) \cdot \sigma(f) \cap \sigma(B)=\emptyset
$$

then there exists a unique solution $x_{f}$ to Eq. (3.4) such that $\sigma\left(x_{f}\right) \subset \sigma(f)$.

In the above theorem and corollary, if $f$ is almost periodic, then so is the unique solution $x_{f}$. Below we will use the following notation $\sigma_{A}(B):=\left\{\lambda \in \mathcal{C}: \nexists(\lambda A-B)^{-1} \in L(\mathbb{X})\right\}$. With this notation we have

Theorem 3.2 If for every $f \in \Lambda(\mathbb{X}) E q$. (3.4) has a unique solution $x_{f}$ such that $\sigma\left(x_{f}\right) \subset \Lambda$, then $\Lambda \cap \sigma_{A}(B)=\emptyset$.

Theorem 3.3 Let $x_{f}$ be a bounded solution to Eq. (3.4). Moreover, assume that $\sigma_{\Gamma, A}(B) \backslash \sigma(f)$ is closed, and that $\sigma(f)$ is countable, and $\mathrm{X}$ does not contain co. Then, there exists an almost periodic solution $w$ to $E q$. (3.4) such that $\sigma(w) \subset \sigma(f)$. 
3.3. Some classical inequalities for derivatives in Banach spaces generated by convex and concave functions

\section{Ha Huy Bang}

Hanoi Institute of Mathematics

In this paper we prove some classical inequalities for derivatives such as inequalities of Landau-Kolmogorov, Bohr, Bernstein for Banach spaces generated by convex and concave functions 


\title{
3.4. Knowledge Discovery and Data Mining Approach to Computational Biology
}

\author{
Ho Tu Bao \\ School of Knowledge Science \\ Japan Advanced Institute of Science and Technology
}

Knowledge Discovery and Data Mining (KDD) has been merged as a rapidly growing interdisciplinary field which merges together databases, statistics, machine learning and related areas in order to extract useful knowledge from large volumes of data. KDD has shown to be a promising approach to computational biology.

This lecture consists of four parts: (1) The main concepts and methods of KDD; (2) the KDD approach to computational biology and challenges; (3) Some results of our research group on $\mathrm{KDD}$; (4) A real-world application of KDD in computational biology: knowledge discovery from stomach cancer data. 


\title{
3.5. On the Stabilizability of Control Systems with Multiple Continuous Delays
}

\author{
Nguyen Sinh Bay \\ nsbay2001@yahoo.com
}

This report presents a result on the stabilizability of a class of control systems, which are described by functional differential equations with time-varying linear perturbations.

The control laws at moment $t$ are constructed, by using the retarded information of the systems on some time-intervals: $\left[t-h_{i}, t\right] ; h_{i}>0, i=1, \cdots, r$

In the report a new sufficient condition of stabilization of the systems is derived. This condition is given by the inequalities on the parameters of the systems and design parameter of the control law.

To establish the main result we use the later generalized Lyapunov operator equation

$$
Q^{\prime}(t)+A^{T}(t) Q(t)+Q(t) A(t)=-P(t)
$$

for the nonautonomous homogeneous system $X^{\prime}(t)=A(t) X(t)$. We also use the particular form of the Lyapunov functional $V\left(t, X_{t}\right)$ on $R^{+} \times C\{-h, 0\}$, which was found by us for the aforementioned systems. 


\title{
3.6. Generalized Collocation RKN Methods
}

\author{
Nguyen Huu Cong \\ Faculty of Mathematics, Mechanics and Informatics \\ HanoiUniversity of Science \\ 334 Nguyen Trai, Thanh Xuan, Hanoi, Vietnam
}

We propose and investigate a very general family of RKN methods for the numerical solution of initial-value problems (IVPs) for systems of special second-orderdifferential equations $y^{\prime \prime}(t)=f[t, y(t)]$. We give not only a generalization of the classical (polynomial) collocation and other recently developed functional-fitting RKN methods but also provide a general stronger result on the order of accuracy for the resulting Generalized Collocation RKN (GRKN) methods (see e.g., Houwen, Sommeijer and Cong, BIT 31 (1991) 469-481; Paternoster, Appl. Numer. Math. 28 (1998) 401-412; Ozawa, Japan J. Indust. Appl. Math. 16 (1999) 25-46; Coleman and Duxbury, J. Comput. Appl. Math. 126 (2000) 47-75). We have theoretically proved that a GRKN method has the same order of accuracy as the classical (polynomial) collocation RKN method. 
3.7. On the recursive sequence $x_{n+1}=\left(A x_{n}+B\right) /\left(x_{n}+a x_{n-1}+b\right)$

Dang Vu Giang

Hanoi Institute of Mathematics

P.O. Box 631, Bo Ho, 10000 Hanoi, Vietnam

E-mail: dvgiang@hanimath.ac.vn

G. Ladas has conjectured that the above positive sequence is convergent for all positive parameters $a, b, A, B$. We prove that this conjecture is true with small restriction on these parameters.

AMS Subject Classification (2000): 39A10

Key words and pharases: rational recursive sequences, global asymptotic stability. 
3.8. Regularization of a nonlinear integral equation of gravimetry

Nguyen Vu Huy ${ }^{1}$, Vo Thi Thanh Nhieu ${ }^{2}$, Chu Van Tho ${ }^{3}$, Dang Dinh Ang ${ }^{4}$

Consider the problem

$$
\int_{0}^{1} \frac{H-\sigma(\xi)}{(x-\xi)^{2}+(H-\sigma(\xi))^{2}} d \xi=f(x)
$$

where $f$ is a given function. We make the following assumption on the solution $\sigma(\xi)$

$$
0<\sigma(\xi)<\alpha<H \text {, where } H \text { and } \alpha \text { are positive constants. }
$$

The problem arises in gravimetry. A two dimensional analogue of Eq. (1) is-also considered. In this paper, we approximate (1) by a linear equation and give an estimate of the error between the exact solution of (1) and that of a regularized linear equation. The two-dimensional case is treated in a similar way.

\section{References}

[1] D. D. Ang, N. V. Nhan and D. N. Thanh, A nonlinear integral equation of gravimetry. Uniqueness and approximation by linear moments, Vietnam J. Math. 27 (1999), 61-67.

[2] D. D. Ang, D. N. Thanh and V. V. Thanh, Indentification of mass inhomogeneity surface gravity anomalies, Geophysical J. International, The Knopoff Volume, Blackwell Science Oxford, UK, 143 (2000), 1-4.

\footnotetext{
${ }^{1}$ Vietnam National University, Ho Chi Minh City

${ }^{2}$ Tay Ninh College of Education

${ }^{3}$ University of Medicine and Pharmacy

${ }^{4}$ Victnam National University, Ho Chi Minh City
} 
3.9. Newmark's method and discrete energy applied to resistive MHD equation

Takashi Kako, and Fumihiro Chiba, The University of Electro-Communications, Chofu, Japan

For the second order time evolution equation with a general dissipation term, we introduce a recurrence relation of Newmark's method. Deriving an energy inequality from this relation, we obtain the stability and the convergence theories of Newmark's method. Next we take up resistive MHD equation as an application of Newmark's method. We introduce a discrete energy of the solution derived from the above energy inequality. We investigate this quantity using numerical experiments. 
3.10. Surface temperature determination from Borehole measurement: Formulation as a moment problem

Tran Thi Le

Vietnam National University, Ho Chi Minh City

We consider the problem of determining the temperature on surface of a half-plane from temperature measured at a sequence of points on a horizontal line below the surface. The problem, formulated as a moment problem, is regularized with error estimates . given. 


\title{
3.11. The improved sweep method for solving nonself-adjoint boundary value problems
}

\author{
Vu Hoang Linh \\ Faculty of Mathematics, Mechanics, and Informatics \\ University of Science, Hanoi National University \\ e-mail:vhlinh@hn.vnn.vn
}

Abstract. Consider the homogeneous boundary value problem for a second order linear differential equation

$$
\begin{aligned}
& y^{\prime \prime}(x)+q(x, \lambda, \alpha) y(x)=0, \quad x_{1} \leq x \leq x_{2} \\
& a_{1} y^{\prime}\left(x_{1}\right)-b_{1} y\left(x_{1}\right)=0 \\
& a_{2} y^{\prime}\left(x_{2}\right)-b_{2} y\left(x_{2}\right)=0
\end{aligned}
$$

Here $\alpha$ is a given parameter and $\lambda$ is a required spectral parameter; $a_{i}, b_{i}(i=1,2)$ are given complex numbers $\left(\left|a_{i}\right|^{2}+\left|b_{i}\right|^{2}>0 ; i=1,2\right)$; Function $q(x, \lambda, \alpha)$ is supposed to be continuous. We are concerned in the computation of eigenvalues (EV-s) and eigenfunctions (EF-s) of a discrete spectrum, which also depends on the parameter. The sweep method that has been proposed previously by Abramov A.A., Konyukhova N.B. et.al. will be improved with special respect to differential equations possessing singularities and large parameters. Based on the Liouville-Green (JWKB) approximation of EF-s, we suggest modifications in the definition of sweep functions and in the construction of sweeping equations. Our aim is the efficient computation of highly oscillatory and(or) rapidly changing functions. When applying the method to singular problems, the stability of numerical integration is provided in the neighbourhood of singular points. Issues on the implementation are also discussed in this contribution. The efficiency of our method is demonstrated by some numerical experiments.

Keywords: Nonself-adjoint boundary value problems, Singular differential equations, Sweep method, Liouville-Green (JWKB) approximation 


\subsection{Some extensions and applications of Gronwall-Bellman's Lemma}

Nguyen Dinh Phu, Hoang Thanh Long

Department of Mathematics and Informatics

Vietnam National University, Ho Chi Minh City

The Gronwall-Bellman's Lemma plays a very important role in the study of the Qualitative Theory of Ordinary Differential Equations (ODE's).

Beside Gronwall-Belman's Lemma, many authors, for example Brezis, Bihari,..., have expansions.

This report has investigated two problem: Expensions and applications of this important lemma. The special new results are given by Lemmas $6,8,9$ and stability conditions of systems which likes control system. 


\subsection{Krylov W-methods for large stiff ODE systems}

H. Podhaisky, R. Weiner, University of Halle, Germany, \{podhaisky,weiner\}@mathematik.uni-halle.de

B.A. Schmitt, University of Marburg, Germany, schmitt@mathematik.uni-marburg.de

We consider linearly implicit one- and two-step W-methods for the MOL solution of reaction-diffusion problems in 2D, where we use Krylov methods to solve the linear algebraic systems in the stages. One-step W-( or ROW-)methods are frequently used for stiff systems. They are characterized by an easy implementation (also for variable stepsizes) and good linear stability properties (A-, L-stable). Using a special multiple Arnoldi algorithm we have constructed Krylov-W-methods, which guarantee the order of the underlying method with relatively low Krylov dimensions independent of the problem size. Parallel two step-W-methods allow the parallel computation of the $s$ stages. They are especially designed for parallel machines with few processors. The construction of methods with stage order $s$ is possible. There are A- and L-stable methods (for constant stepsizes). Due to their high stage order there is (in contrast to one-step W-methods) in general no order reduction for very stiff problems. However; stability investigations for variable stepsizes are difficult and the methods show often a sensitive dependence of stability and error constants on stepsize changes. The main work of the computation of the stage values can be done in parallel if Krylov methods are used for the solution of the linear systems. The speedup is close to s for expensive right hand sides. The parallelization is done automatically in the code, a user needs to provide only the right-hand side (as usual for sequential methods). The performance of the two types of W-methods on MOL-problems is discussed and compared with the efficient sequential code VODPK. 


\subsection{Black-Scholes PDE: A Finance Application}

\section{Vuong Hoang Quan}

Ecole de Commerce Solvay (Bruxelles)

The field of financial economics has developed significantly since the first half of the century. Its fast growth inherited greatly from the development in theories. Today, the financial markets deal with thousands of financial products, worth hundreds of billions of dollars. Such markets demand the fairness in transaction through computing accuracy. Naturally, the financial economics has emerged as a hybrid field of social science and computing methods, or econophysics as put by Mantegna et al (1999). This communication aims to articulate a hallmark development in finance since the 1970 s, the Black-Scholes partial differential equation (B-S PDE). The B-S PDE represents a spectacular application of a seemingly unrelated scientific field, physics, in today's financial world. 


\subsection{Analysis of a Chemostat Model for Bacteria and Bacteriophage}

\section{Edoardo Beretta *, Hirotatsu Sakakibara ${ }^{* *}$ and Yasuhiro Takeuchi**}

\footnotetext{
*Istituto di Biomatematica, Universita di Urbino, I-61029 URBino, Italy

**Department of Systems Engineering, Faculty of Engineering, SHizuoka UNIVERSITY, HAMAMATSU 432-8561, JAPAN
}

The dynamical properties of the time delayed chemostat model described by

$$
\begin{aligned}
\dot{r} & =\rho(C-r)-\phi_{1} n_{1}-\dot{\phi}_{2} n_{2} \\
\dot{n}_{1} & =n_{1}\left(\phi_{1} / e_{1}\right)-\rho n_{1}-\gamma_{1} n_{1} p \\
\dot{n}_{2} & =n_{2}\left(\phi_{2} / \epsilon_{2}\right)-\rho n_{2} \\
\dot{p} & =b_{1} \gamma_{1} e^{-\rho l_{1}} n\left(t-l_{1}\right) p\left(t-l_{1}\right)-\rho p-\gamma_{1} n_{1} p
\end{aligned}
$$

are considered. Here $r(t), n_{1}(t), n_{2}(t)$, and $p(t)$ are a concentration of the resource, the densities of two bacteria and bacteriophage, respectively. Further, $\rho$ is the rate of flow through the chemostat, $C$ is the input concentration of the resource, $\gamma_{1}$ is the attack constant of phage to the first bacteria, $\epsilon_{i}$ is the bacteria's consumption rate of the resource, $l_{1}$ is the latent period (the time delay between the attack by a phage on the first bacteria and the resulting reproduction of new phages) and $b_{1}>1$ is the reproduction rate of the phage from the infected first bacteria. The $\phi_{i}$ is the bacteria's taking up rate of the resource and satisfies $\phi_{i}(0)=0$ and increasing in $r>0$.

Note that the first bacteria is assumed to be sensitive to predation of the phage but the second is immune to predation. Some experimental data show that two bacteria (the first is resident and the second is a mutant) and phage can coexist. First, we consider the boundedness of the solutions of (1). Second, using the geometric stability switch criterion in the delay differential system with delay dependent parameters, we present the local asymptotic stability of nonnegative equilibria. We further show that the coexistence is possible for short latent period and for large reproduction rate of the phage. 
Digitized by GoOgle 


\subsection{Permanence of host,parasitoid systems}

\section{Ryusuke Kon and Yasuhiro Takeuchi}

Department of Systems Engineering, Faculty of EngineEring, Shizuoka UNIVERSITY, HaMAMATSU 432-8561, JAPAN

The interactions of a host and a parasitoid are modeled by difference equations because of their non-overlapping generations (see Hassell, 1978). Even one dimensional difference equations have very complicated solutions (see May \& Oster, 1976). It is difficult to predict an asymptotic behavior of such solutions, so that we examine the condition for permanence, which requires that all solutions eventually enter and remain in a region with a non-zero distance from the boundary.

Permanence of difference equations of Lotka-Volttera type was investigated by Hofbauer et al. (1987). By using the technique in Hofbauer et al. (1987) and the theory of an average Liapunov function (Hutson, 1984), we examine the condition for permanence of the following 2-host 1-parasitoid system:

$$
\left\{\begin{aligned}
u_{1}(t+1)= & r_{1} u_{1}(t) \exp \left[-\left(u_{1}(t)+u_{2}(t)\right)-v(t)\right] \\
v(t+1)= & c_{1} u_{1}(t)(1-\exp [-v(t)]) \\
& +c_{2} u_{2}(t)(1-\exp [-\alpha v(t)]) \\
u_{2}(t+1)= & r_{2} u_{2}(t) \exp \left[-\beta\left(u_{1}(t)+u_{2}(t)\right)-\alpha v(t)\right] .
\end{aligned}\right.
$$

A local stability analysis of a positive equilibrium of system (1) was carried out by Comins and Hassell (1976). The sufficient condition for permanence of system (1) in the absence of $u_{1}$ or $u_{2}$ was obtained by Kon and Takeuchi (preprint).

\section{References}

1. Comins, H. N. and Hassell, M. P. (1976). Predation in Multi-prey Communities. Journal of Theoretical Biology 62, 93-114.

2. Hassell, M.P. (1978). The dynamics of arthropod predator-pray systems. Princeton University Press Princeton, NJ.

3. Hofbauer, J., Hutson, V. and Jansen, W. (1987). Coexistence for systems governed by difference equations of Lotka-Volterra type. J. Math. Biol. 25, 553-570.

4. Hutson, V. (1984). A theorem on average Liapunov functions. Monash Math. 98, 267-275.

5. Kon, R. and Takeuchi, Y. (preprint). Permanence of host-parasitoid systems.

6. May, R. M. and George Oster, F. (1976). Bifurcations and dynamics complexity in simple ecological models. Am. Nat. 110, 573-599. 


\title{
3.18. Nonlinear Functional Integral Equation and Its Application
}

\author{
Akira Yanagiya \\ Waseda University \\ 3-31-1 Kamishakuzii, Nerima-ku, Tokyo 162-0804, Japan \\ Tel: 81-3-5991-4151 I' $\Lambda$ X81-3-3928-1110 \\ E-mail: yanagiya@mn.waseda.ać.jp
}

In this talk we consider the next functional integral equation.

$$
\begin{aligned}
x(t)= & \int_{0}^{t} k(t-s, t ; x) y(s) d s+\int_{0}^{\infty} L(t, s ; x) \dot{\varphi}(s) d s \\
y(t)= & \int_{0}^{t} \beta(t-s, x(t)) k(t-s, t ; x) y(s) d s \\
& +\int_{0}^{\infty} \beta(t+s, x(t)) L(t, s ; x) \varphi(s) d s
\end{aligned}
$$

We usually treat this type integral equation when we investigate the following nonlinear population model.

$$
\begin{aligned}
& \frac{\partial n}{\partial a}+\frac{\partial n}{\partial t}+\mu(a, N(t)) n(a, t)=0, \quad a>0,0<t<T \\
& n(0, t)=\int_{0}^{\delta} m(a, N(t)) n(a, t) d a, \quad 0<t \leq T, \\
& n(a, 0)=\varphi(a), \quad a \geq 0 .
\end{aligned}
$$

In this conference, we may propose the several existence theorems of solutions for the integral equation and Kneser type theorem under the following hypotheses.

$$
\begin{aligned}
& \beta \in C\left(R^{+} \times R\right) \\
& k(t, s ; x): \text { cont.on }[0, T] \times .[0, T] \times \Sigma \\
& L(t, s ; x): \text { cont.on }[0, T] \times R^{+} \times \Sigma \\
& |L(t, s ; x)-1| \longrightarrow 0 a . s T \rightarrow 0, \\
& \text { on } 0 \leq t, s \leq T, x \in \Sigma
\end{aligned}
$$


